\title{
Effects of a structured 20-session slow-cortical- potential-based neurofeedback program on attentional performance in children and adolescents with attention-deficit hyperactivity disorder: retrospective analysis of an open-label pilot-approach and 6-month follow-up
}

This article was published in the following Dove Press journal:

Neuropsychiatric Disease and Treatment

2 March 2017

Number of times this article has been viewed

Johanna S Albrecht, ${ }^{1-3}$ Sarah Bubenzer-Busch, ${ }^{1,2}$ Anne Gallien, ${ }^{1,4}$ Eva Lotte Knospe, 1,2 Tilman J Gaber, ${ }^{1,2,5}$ Florian D Zepf $^{1,2,6,7}$

'Clinic for Child and Adolescent Psychiatry, Psychosomatics and Psychotherapy, RWTH Aachen University, Aachen, ${ }^{2}$ JARA Translational Brain Medicine, Aachen \& Jülich, ${ }^{3}$ Center for Pediatrics and Adolescent Medicine Elisabeth Hospital Rheydt, Mönchengladbach, ${ }^{4} \mathrm{Clinic}$ for Neurology, Medical Center City Region Aachen, Würselen, ${ }^{5}$ NeuroCare Group, Munich, Germany; ${ }^{6}$ Centre and Discipline of Child and Adolescent Psychiatry, Psychosomatics and Psychotherapy, School of Paediatrics and Child Health \& School of Psychiatry and Clinical Neurosciences, Faculty of Medicine, Dentistry and Health Sciences, The University of Western Australia, ${ }^{7}$ Department of Health in Western Australia, Specialised Child and Adolescent Mental Health Services, Perth,WA, Australia
Correspondence: Florian D Zepf

Centre and Discipline of Child and Adolescent Psychiatry, Psychosomatics and Psychotherapy, The University of Western Australia, 35 Stirling Highway (M56I), Crawley, Perth, WA 6009, Australia

Email florian.zepf@uwa.edu.au
Objective: The aim of this approach was to conduct a structured electroencephalography-based neurofeedback training program for children and adolescents with attention-deficit hyperactivity disorder (ADHD) using slow cortical potentials with an intensive first (almost daily sessions) and second phase of training (two sessions per week) and to assess aspects of attentional performance.

Patients and methods: A total of 24 young patients with ADHD participated in the 20 -session training program. During phase I of training ( 2 weeks, 10 sessions), participants were trained on weekdays. During phase II, neurofeedback training occurred twice per week ( 5 weeks). The patients' inattention problems were measured at three assessment time points before (pre, T0) and after (post, T1) the training and at a 6-month follow-up (T2); the assessments included neuropsychological tests (Alertness and Divided Attention subtests of the Test for Attentional Performance; Sustained Attention Dots and Shifting Attentional Set subtests of the Amsterdam Neuropsychological Test) and questionnaire data (inattention subscales of the so-called Fremdbeurteilungsbogen für Hyperkinetische Störungen and Child Behavior Checklist/4-18 [CBCL/4-18]). All data were analyzed retrospectively.

Results: The mean auditive reaction time in a Divided Attention task decreased significantly from T0 to T1 (medium effect), which was persistent over time and also found for a T0-T2 comparison (larger effects). In the Sustained Attention Dots task, the mean reaction time was reduced from T0-T1 and T1-T2 (small effects), whereas in the Shifting Attentional Set task, patients were able to increase the number of trials from T1-T2 and significantly diminished the number of errors (T1-T2 \& T0-T2, large effects).

Conclusion: First positive but very small effects and preliminary results regarding different parameters of attentional performance were detected in young individuals with ADHD. The limitations of the obtained preliminary data are the rather small sample size, the lack of a control group/a placebo condition and the open-label approach because of the clinical setting and retrospective analysis. The value of the current approach lies in providing pilot data for future studies involving larger samples.

Keywords: SCP, neurofeedback, ADHD, children, adolescents, attention 


\section{Introduction}

Attention-deficit hyperactivity disorder (ADHD) is a common psychiatric disorder in children and adolescents, which comprises the three-core symptom domains of increased hyperactivity, impulsivity and inattentiveness. In Germany, prevalence rates of $\sim 4.8 \%$ in children and adolescents have been reported, ${ }^{1}$ which is consistent with international prevalence rates. ${ }^{2}$ However, with regard to the diagnostic criteria in the Diagnostic and Statistical Manual of Mental Disorders (DSM)-5, published by the American Psychiatric Association (APA), ${ }^{3}$ ADHD can persist into late adolescence and adulthood. The DSM-5 has been subject to significant modifications compared with the DSM-4 (such as the number of symptoms required, duration of symptoms, age of onset and severity of outcome). Regarding outcome, ADHD in childhood can have a negative impact on educational and occupational achievements, social and occupational functioning, divorce rates, the rates of other comorbid psychiatric disorders and substance abuse in later adult life. ${ }^{4}$ As far as strategies for intervention are concerned, a multimodal treatment approach for children with ADHD is widely accepted. Several guidelines currently suggest a combination of education about the disorder for the parents, patients and teachers, training of parents and family, interventions in school settings and cognitive and behavioral therapeutic interventions, along with psychopharmacological treatment strategies. ${ }^{5,6}$ Medications commonly used in the treatment of ADHD, such as methylphenidate, atomoxetine and amphetamines, can have numerous side effects, such as reduced growth and appetite, suicidal ideation, cardiac arrhythmia and insomnia. In addition, clinical samples exhibit significant rates of nonresponders to medication. ${ }^{7}$ Thus, alternative strategies for intervention and treatment are needed and are the subject of translational neurobiological research.

One alternative intervention of interest for the treatment of ADHD is neurofeedback (NF), which can be performed using an electroencephalography (EEG) and was first described by Lubar and Shouse, ${ }^{8}$ and (although largely experimental and even more explorative) in an functional magnetic resonance imaging (fMRI) environment. ${ }^{9} \mathrm{NF}$ training aims to enable the subject to regulate brain activity related to ADHD symptoms in real time, such as brain regions associated with attentional processes. NF training is often combined with a behavioral therapeutic approach, which is based on operant conditioning so that the patient learns to achieve better self-control. NF is also used in the treatment of other psychiatric and neurological disorders, such as migraines,${ }^{10}$ tic disorders ${ }^{11}$ and epilepsy. ${ }^{12}$
There are some unspecific EEG changes observable in patients with ADHD. As described by Lubar, ${ }^{13}$ increased theta (4-8 Hz) activity and decreased beta $(13-21 \mathrm{~Hz})$ activity have been identified in patients with ADHD, which has resulted in an NF-based training approach that aims to teach children with ADHD to decrease the theta/beta ratio in fronto-central brain regions. These areas are important for attention processes and for controlling different behaviors. ${ }^{14}$ Another potential approach of NF is the training of the so-called slow cortical potentials (SCP), which was first studied by Elbert et $\mathrm{a}^{15}$ and the research team of Niels Birbaumer. ${ }^{16}$ SCPs are slow changes in brain potentials with frequencies below 1 and $2 \mathrm{~Hz}$ that can be seen in preparation for a specific event, for example, a certain motor or cognitive response. In particular, SCPs are influenced by the activity of large cortical neuron assemblies, and they are believed to play a decisive role with regard to the cortical control of lower brain regions (such as the striatum) in cortico-striato-thalamo-cortical (CSTC) feedback loops. SCPs in the negative direction are believed to be caused by the depolarization of large cortical assemblies and exhibit a lower excitation threshold, whereas SCPs in the positive direction are believed to be caused by repolarization of these cell assemblies and thus create a higher excitation threshold. ${ }^{17,18} \mathrm{SCP}$-based NF training has the goal to create SCP shifts in the negative direction and thus to reduce the excitation thresholds of large cortical cell assemblies. According to Rockstroh, Elbert et al, ${ }^{19}$ children with attention problems cannot control their SCPs as well as healthy children. Heinrich et al compared children with ADHD who had participated in an SCP-based NF training program with children on a waiting list for treatment and identified a significant reduction in ADHD symptoms and an increase in contingent negative variation (CNV), a typical $\mathrm{SCP}$, in the SCP training group. ${ }^{20}$

Comparisons of the theta/beta-related and SCP-based NF training in children with ADHD have identified significant improvements in cognition and behavioral characteristics for both training groups. ${ }^{21-23}$ In a recent study, Gevensleben et $\mathrm{al}^{24}$ demonstrated that SCP-based NF training in children with tic disorders reduced the accompanying ADHD symptoms, such as hyperactivity and impulsivity, better than NF training that aimed to target the theta/beta ratio. However, it must be noted that a recent review article on the effects of NF as a treatment approach for ADHD suggests further research on psychological approaches on ADHD such as NF. ${ }^{25}$

A number of studies have been conducted to date, which have first demonstrated, in principle, positive results for NF training in children with ADHD. To date, most studies have 
used between $40^{26}$ and $25^{20}$ training sessions, which must be considered a significant effort for the patients and their families. A lower number of training sessions has been suggested to potentially have smaller effects, especially regarding the symptoms of inattention as outcome measures. ${ }^{27}$ Although Arnold et $\mathrm{al}^{26}$ suggest that 30 training sessions should most likely be sufficient according to parental ratings, they are not certain if 30 sessions are able to result in a maximum therapeutic effect. The more practical positive aspects of shorter NF training programs are clear, such as a potential cost reduction and the training of additional patients during a certain time span, as well as a lower effort for patients and their families. Here, we aimed to explore the effects of a structured 20-session-based SCP-related NF training within an explorative open-label approach in a sample of children and adolescents with ADHD. To enable a steeper slope of the learning curve as far as SCP-related regulative capacity within the outlined CSTC loops was concerned, we divided the training program into two phases with an equal number of sessions, with the first phase comprising a higher number of training sessions (intensified training). The first phase consisted of 10 sessions, and NF training sessions were administered on a daily basis on working days over a period of 2 weeks (five NF training sessions per week). The second phase comprised a period of 5 weeks with two sessions per week.

\section{Patients and methods \\ Participants}

The training program was conducted at the Clinic for Child and Adolescent Psychiatry, Psychosomatics and Psychotherapy,
RWTH Aachen University, Aachen, Germany. The participants were recruited through the Child and Adolescent Psychiatric clinic and information materials were distributed by local children and adolescent psychiatrists and ADHD support services. A waiver from the Ethics Committee of the Faculty of Medicine of RWTH Aachen University (Aachen, Germany) was obtained to analyze the data of the clinical NF treatment program retrospectively after the completion of the program. All patients and their parents agreed to participate in the NF training program. A total of 24 children and adolescents with ADHD aged 7-17 years (mean $=11.47$ years; standard deviation $[\mathrm{SD}]=3.02$ years) participated in this structured NF training program. Prior to the pre-training assessment, all participants were diagnosed with ADHD by their local child and adolescent psychiatrists according to DSM-4 criteria. All patients had an IQ above 80 as assessed with different standardized tests such as the German editions of the Culture Fair Intelligence Test (CFIT), ${ }^{28}$ the Wechsler Intelligence Scale for Children (WISC IV) ${ }^{29}$ or the Kaufman Assessment Battery for Children (K-ABC). ${ }^{30}$ Information on this testing was provided by the child and adolescent psychiatrists in charge of patient treatment. Ten of the 24 trainees took various doses of methylphenidate. The participants were free of psychostimulants on the days of psychological testing and NF training. Children and adolescents treated with atomoxetine did not participate in the training program. The trainees with common comorbidities related to ADHD, such as conduct disorder (CD) or dyslexia, were not excluded. The numbers and comorbidities of the participants are shown in Table 1. A total of 24 patients participated in a pre-training assessment, but because of computer problems, the data of only

Table I Characteristics of the study sample

\begin{tabular}{|c|c|c|c|}
\hline Characteristics & $\begin{array}{l}\text { pre-training sample } \\
(\mathrm{N}=24)\end{array}$ & $\begin{array}{l}\text { Post-training } \\
\text { sample }(\mathrm{N}=22)\end{array}$ & $\begin{array}{l}\text { Follow-up sample } \\
(\mathrm{N}=13)\end{array}$ \\
\hline Age (years; months) (range) & $11.47 \pm 3.02(7-17)$ & $11.27 \pm 2.82(7-17)$ & $11.78 \pm 2.82(8-18)$ \\
\hline Gender (boys; girls) & I8; 6 (75\%; 25\%) & $16 ; 6$ (72.73\%; 27.27\%) & $9 ; 4$ (69.20\%; 30.78\%) \\
\hline \multicolumn{4}{|l|}{ DSM-4 subtype } \\
\hline Combined type & 21 & 19 & 12 \\
\hline Inattentive type & 3 & 3 & 1 \\
\hline Drug naïve & 14 & 12 & 6 \\
\hline \multicolumn{4}{|l|}{ Comorbidities } \\
\hline $\mathrm{CD}$ & 9 & 7 & 5 \\
\hline Depression & I & & \\
\hline Developmental disorder of scholastic skills & 5 & 3 & 3 \\
\hline Language disorder (unspecified) & 1 & I & 1 \\
\hline Obesity & I & I & \\
\hline PTSD & I & & \\
\hline
\end{tabular}

Notes: The number, age, gender and diagnosis of the participants at the three time points of assessment (before NF training = pre; after the 20 th session = post; 6 months after the last training session = follow-up) and the number of drug-naïve trainees and the participants' comorbidities are given.

Abbreviations: CD, conduct disorder; DSM, Diagnostic and Statistical Manual of Mental Disorders; NF, neurofeedback; PTSD, posttraumatic stress disorder. 
23 participants were analyzed. There was one dropout prior to the post-training assessment, and the data of one patient were not recorded. All participants were invited to a follow-up assessment, but only 13 participants fully completed this assessment. Moreover, the symptom-related questionnaires (which served as pre- and post-behavioral assessments) were not all completed and returned.

\section{NF training regime}

The 7-week 20-session training program first consisted of an intensive training phase (phase I, 2-week duration) and with five 1-hour NF training sessions per week. After completion of phase I, during the following 5 weeks, participants received two NF training sessions per week (phase II). The schedule of the structured NF training program is provided in Figure 1.

The training was conducted in a separate and quiet room, and the participants were advised by a personal NF coach. An EEG device, a CE-certified NF system (Thera Prax ${ }^{\circledR}$, Version 2.3.0; Eldith/NeuroConn, Ilmenau, Germany), was used, which was equipped with a Thought-Translation Device (TTD) software. ${ }^{31}$ The feedback signal was assessed and calculated from the $\mathrm{Cz}$ electrode position, which was referenced by the left mastoid. To reduce artifacts produced by subject eye movements, a real-time electrooculogram (EOG) was recorded simultaneously, using four electrodes (over and under the left eye to detect vertical movements and lateral to both eyes to assess horizontal eye movements). Eye movements were analyzed in real time and were removed from the feedback signal so that the participants were presented with an SCP signal free of eye movement artifacts. A trial was not validated and repeated when the artifacts were $\pm 200 \mu \mathrm{V}$ in the EEG channel and $\pm 800 \mu \mathrm{V}$ in the EOG channel. In addition to the artifact control, an EOG correction factor was implemented when the EEG and EOG were of the same \pm sign. A trial was canceled when the amplitude of the EOG activity surpassed the EEG activity; however, when the EEG activity exceeded the EOG activity, the EEG amplitude was corrected by $15 \%$ of the mean EOG amplitude, which is a standard procedure. ${ }^{32,33}$ The sampling rate (EEG and EOG) was set at $128 \mathrm{~Hz}$. The SCP measurement for one trial was based on that particular trial.

A training session not only consisted of computerbased attention-demanding NF tasks in which the subjects had to up- or downregulate their own SCP activity but also included the development of individual strategies to complete these tasks. For example, trainees were advised to think of situations associated with positive emotions for negative SCPs (such as roller coaster rides or scoring a goal in soccer), and relaxing activities (such as dreaming or lying still in bed) for positive SCPs. The suggested strategies were used only as a starting point, and patients were encouraged to find individual strategies to create negative and positive SCPs. These strategies were documented in each training session and changed or adapted by the trainee if the strategy did not lead to SCP changes in the desired direction. The first suggested strategies might have served as a placebo, but all further strategies were chosen by the trainee and were thus not subject of or related to a placebo effect. Negative SCPs (activation) were related to making the visual correlate of SCP activity move upward above a threshold line that signified $33 \%$ of negative SCPs, and positive SCPs (deactivation) were related to making the visual correlate of SCP activity move downward underneath a threshold line that signified $33 \%$ of positive SCPs. Threshold lines were included to prevent false-positive results in SCP changes. Positive SCPs, as well as negative SCPs were included to allow the trainees a better differentiation between deactivation and activation and to learn to differentiate and to control the switch between relaxation and the activation of cognitive resources. Successful differentiation between these two states is important for young people with ADHD to be able to manage attentionrelated tasks.

The patients received positive feedback (a rewarding stimulus, eg, an image of a sun) on the computer screen when they managed to successfully regulate as intended (upward or downward as per instruction; Figure 2) within the trial (a more detailed description of the physiological regulation criteria set is given in the following sections). Each session

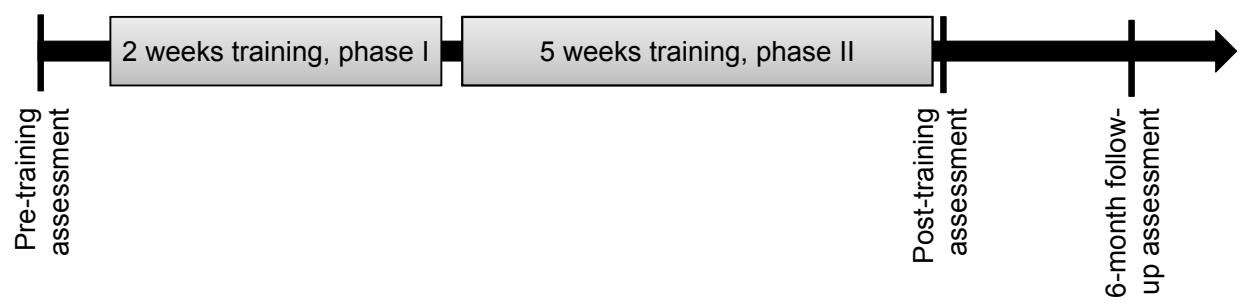

Figure I Training regime.

Note: An overview of the training regime and assessment points is shown. 

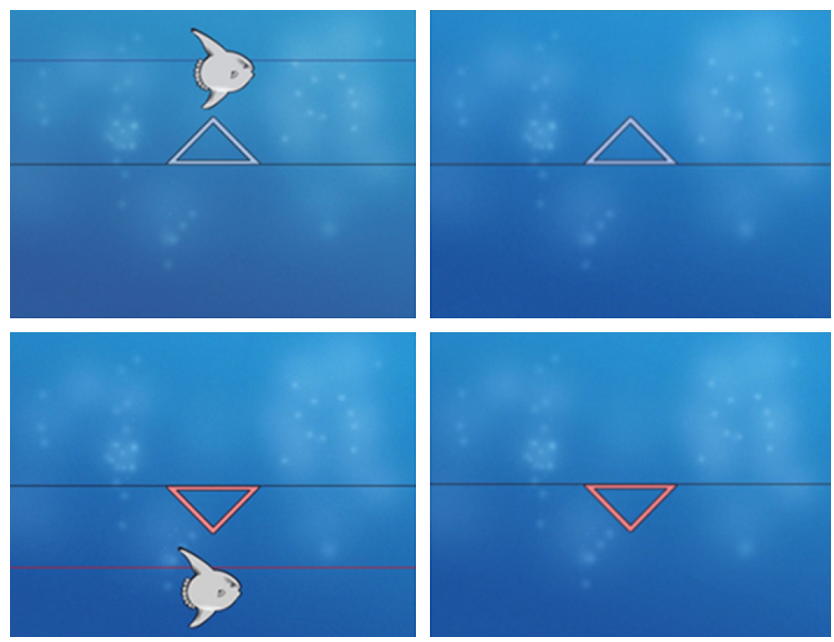

Figure 2 Participant's monitor during feedback and transfer trials (images provided with kind permission by NeuroConn, Ilmenau, Germany).

Notes: The screenshots of the participant's monitor during feedback period I (on the left with feedback, on the right during transfer trials) are shown. On the upper graphics, the feedback monitor shows the screenshots during a negativity trial (blue arrow pointing upward), the bottom black line indicates the baseline and the upper blue line marks the cutoff line. The goal is to move the stimulus (fish) above the upper line. A positivity trial is shown on the two lower graphics (red arrow is pointing downward).

comprised three runs of NF training, with intermissions of several minutes duration in-between runs. One run consisted of a different number of trials, which increased with the number of training sessions completed by the trainee throughout the program. The number of trials and runs are provided in Table 2.

A trial was 8 seconds long and could be divided into three parts. During the first part ( 2 seconds), participants watched a blue screen and a first baseline regarding SCP activity was assessed. Throughout the second part ( 5.5 seconds, feedback period I), the participants viewed an arrow that pointed up- or downward on their feedback monitor. Here, the task was to move a visual correlate of the participants' SCP activity (ie, a fish) in the demanded direction over or under the threshold line by producing negative SCPs compared with the baseline (upward fish movement) or positive SCPs (downward fish movement). The criterion for successful regulation was to achieve a change in SCP regulation for at least 2 seconds duration in the second half of the trial, which meant to successfully change SCP regulation to a degree of $30 \%$ of $80 \mu \mathrm{V}(24 \mu \mathrm{V})$. In the course of the third part (half a second, feedback period II), successful regulation was rewarded with a picture of a shining sun (highly significant change in SCP activity when compared with baseline $=100 \%$ of the abovementioned criterion achieved over 2 seconds) or a clouded sun (significant increase in SCP activity compared with baseline $=80 \%$ of the mentioned criterion achieved over 2 seconds [ $80 \%$ of $30 \%$ of $80 \mu \mathrm{V}=19.2 \mu \mathrm{V}]$ ) in the sense of operant conditioning. When the participants failed to create the changes needed (not achieving the criterion that was set as mentioned earlier), they viewed the blue standardized background screen of the NF training setup of the respective device.

During the transfer trials, which were introduced in session 7 (sequence of administration was randomized in real time between normal feedback trials), participants only viewed the screen with the arrow during feedback period I and received no visual SCP feedback (ie, in the form of a picture of a fish) with the exception of the reward signal in the case of a successfully completed trial. The aim of the transfer trials was to approximate the training to daily life situations. The rationale for using different ratios of negative/positive regulation trials and transfer rates across sessions (Table 2) was related to clinical feasibility and usability, with a focus on activation (negativation) as the clinically most meaningful regulation direction derived from the symptoms observed in young patients with ADHD. Otherwise, the frustration about not clearly visible improvements in terms of successful upregulation of SCP during feedback trials, frustration about unsuccessful regulation of transfer trials and covarying compliance issues, might have led young patients with ADHD to withdraw from the training. Starting from session 10 , participants were supplied with a "transfer card" to practice

Table 2 Training protocol

\begin{tabular}{clllllll}
\hline Session & Algorithm & Trials & FB- & FB+ & TR- & TR+ & Ratio (negative/positive) \\
\hline $\begin{array}{c}\text { Phase I } \\
\text { I-6 }\end{array}$ & SCP_6 min & 32 & 16 & 16 & 0 & 0 & $50 / 50$ \\
7-I0 & SCP_32_8 & 40 & 16 & 16 & 4 & 4 & $50 / 50$ \\
Phase II & & & & & & \\
II & SCP_32_8 & 40 & 16 & 16 & 4 & 4 & $50 / 50$ \\
I2-I5 & SCP_40_20_20 & 40 & 10 & 10 & 10 & 10 & $50 / 50$ \\
I6-20 & SCP_50_30_20 & 50 & 18 & 12 & 12 & 8 & $60 / 40$ \\
\hline
\end{tabular}

Notes: The training protocols for each session and training phases I (five sessions per week, 2 weeks duration) and II (two sessions per week, 5 weeks duration) are shown. The number of trials, number of trials with negative FB (FB-), positive FB (FB+), negative TRs (TR-), positive TRs (TR+), the ratio of negative and positive trials (ratio [negative/positive]) and the percentage of the number of transfer trials, are given.

Abbreviations: FB, feedback; SCP, slow cortical potential; TR, transfer trial. 
their individual regulation-related strategies at home. They were advised to practice their regulation strategies in daily life settings (duration, $\sim 5$ minutes), in particular, in situations in which they needed to be alert and attentive (ie, during school examinations or while doing their school homework) or relaxed (ie, before going to sleep). All patients were provided a homework sheet on which they had to fill in the time and the situation in which they applied their strategies. The participants were also provided a booklet in which they could earn points for each successful session in the sense of a positive reinforcement, which is consistent with common behavioral therapeutic techniques. The transfer cards were introduced as an add-on mechanism to allow practicing NFrelated strategies at home.

\section{Assessment of SCP regulation}

The percentage of correct SCP regulation of all participants was calculated for each session as depicted in Figure 3.
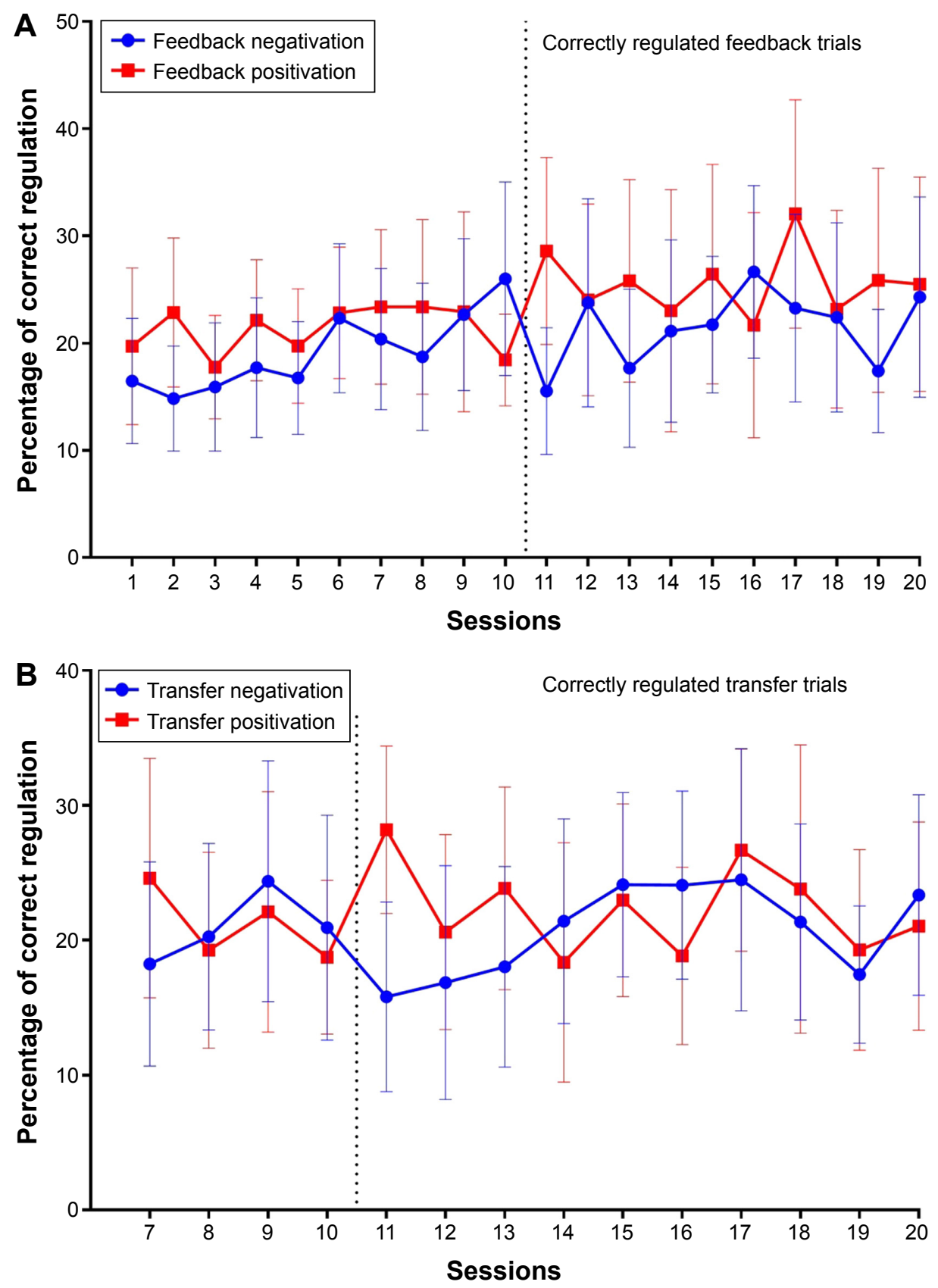

Figure 3 Percentage of correctly regulated negativation and positivation feedback $(\mathbf{A})$ and transfer $(\mathbf{B})$ trials per session.

Notes: The mean percentage of correctly regulated trials per session of all participants is shown. Negativation trials are demonstrated in blue, positivation trials in red. $95 \%$ Confidence intervals are displayed as error bars. The dashed line signifies the change of training regime between phase I (daily sessions) and phase II (sessions twice a week). 


\section{Neurocognitive testing}

As regards the order of neurocognitive tests, all tests were administered (a more detailed description is given in the following sections) in the same order/sequence in terms of a standardized approach. The total time for neurocognitive testing per patient was $\sim 60-75$ minutes (depending on task performance in the last test, which was the Sustained Attention Dots [SAdots] task), and with sufficient breaks between each of the tests. As a consequence, there were no effects of testing schedule or date to be expected.

\section{Assessment of attentional performance}

Attentional performance was assessed using different neuropsychological tests at three different time points: Before the beginning of the training program (pre), after the 20th session (post) and 6 months after the end of training (follow-up). The following neuropsychological tests were completed by the participants: two subtests of the Test for Attentional Performance 1.7 (TAP 1.7), ie, Alertness and Divided Attention. ${ }^{34}$ The TAP is a standardized computerbased test battery, which aims to assess different aspects of attention. During the Alertness test, the participants were asked to react as quickly as possible to a visual stimulus (a white cross that appeared on a black screen) by pressing a key. The test is composed of two parts: one part without and one part with an auditive warning signal to compare intrinsic alertness (without warning signal) to phasic arousal (with a warning signal). The mean response times, SDs and numbers of missed trials were analyzed in this paper as a measure of response time change.

The Divided Attention test aims to assess the ability to simultaneously complete two tasks: one visual task and one auditory task. The participants viewed a black screen with a varying number of moving crosses and were asked to press a key when four of the crosses formed a square. Simultaneously, high- and low-tone sequences were presented in a random order and the participants had to press the key when the same tone occurred twice in a row. The mean response times, SDs and numbers of mistakes/missed trials were analyzed for both conditions in this paper as a measure of Divided Attention between two simultaneously occurring stimuli.

Two subtests of the Amsterdam Neuropsychological Tests (ANTs), such as the SAdots and the Shifting Attentional Set (SSvis), ${ }^{35}$ were performed. The ANT is a computerized test battery that includes 32 response time tasks and is used in both research and clinical settings for a systematic assessment of information processing capacities. During the SAdots task, 600 dot patterns are presented on a monitor in
50 series of 12 trials. Each series was composed of four three-, four-, and five-dot patterns in a pseudorandom sequence. The participants had to react with their dominant hand by pressing a mouse button when they saw a four-dot pattern (compatible response, hit) and to respond to three- or five-dot patterns by pressing the mouse button with their nondominant hand (incompatible response, correct rejection). Inaccurate responses were followed by auditory feedback in the form of a beeping noise. The reaction time for correct responses (hits and correct rejections) and the number of incorrect responses (misses and false alarms), as well as the SD were used as main outcome variables.

The SSvis test is composed of three trials. The participants viewed a horizontal bar, which was composed of 10 white squares on a black screen. In each of the three parts of the test, a colored square moved from square to square in a random order. The participants held a computer mouse in both hands. During part 1, which included 40 task trials, the square was green and the participants were asked to press the mouse key in the direction the square had moved (compatible reaction). In part 2 (40 task trials), a red square appeared and the participants had to press the key opposite to the square movement (incompatible reaction). Part 3 consisted of 80 task trials and demonstrated appearing and disappearing red and green squares in a random order. Green squares had to be followed by a compatible reaction (similar to part 1) and red squares by an incompatible reaction (similar to part 2). The mean reaction times, SDs and number of mistakes were analyzed.

\section{Assessment of ADHD symptoms and psychopathology}

The following questionnaires were completed at the pre-, post- and follow-up time points (duration together with parent interview was between 60 and 90 minutes): the German ADHD rating scale (FBB-HKS) ${ }^{36,37}$ was completed by parents and teachers. The FBB-HKS is a 20-item questionnaire that uses the ICD-10 and DSM-4 criteria to help diagnose hyperkinetic disorders. It comprises three subscores, including inattention, hyperactivity and impulsivity, and a total score. Items can be rated from 0 to 3 (not true - especially true), with respect to symptom existence and challenge. The Child Behavior Checklist/4-18 (CBCL/4-18) German version $^{38,39}$ was completed by the subjects' parents. The $\mathrm{CBCL} / 4-18$ is a frequently used standardized clinical assessment tool to acquire information regarding a child's competencies and behavioral problems. It includes the assessment of the following eight syndrome scales: withdrawal, somatic 
complaints, anxiousness/depression, social problems, thought problems, attention problems, delinquent behavior and aggressive behavior. Because we were primarily interested in the efficacy of NF training on attentional performance and to reduce $\alpha$-errors, only the variables that correlated with attention were included in the analysis, which includes the inattention subscale of the FBB-HKS and the attention subscale of the CBCL.

\section{Data analysis}

The analysis of data was performed using a retrospective approach. The pre- and post-neuropsychological test performances and questionnaire results were compared using two-tailed paired $t$-tests. The normality of the data was verified using the Shapiro-Wilk ${ }^{40}$ and Kolmogorov-Smirnov tests. For data in which the assumption of normality was not validated, the Wilcoxon signed rank test was calculated in addition to the two-tailed paired $t$-test. The Wilcoxon signed rank tests identified similar results compared with the $t$-tests conducted with the not normally distributed data regarding statistical significance; for better comparability, the $t$-tests were used for further interpretation and the calculation of the estimated effect sizes. The level of statistical significance was set at $P<0.05$. The effects of multiple comparisons on the same data set were addressed using a two-tailed $\alpha$-adjustment according to the Bonferroni-Holm procedure (adjusted $P$-values are indicated by $P^{\prime}$ ), and the effect sizes were estimated using Cohen's $d$ values. In the case of a significant outcome, the post- and follow-up results were compared in a similar way to test for the stability in attentional performance changes.

\section{Results}

The data of the pre (T0), post (T1) and follow-up (T2) results of the different tests for the assessment of attentional performance are provided in Tables 3-5.

\section{Alertness}

On a descriptive level, in the Alertness subtest of the TAP, the mean reaction time, SD and phasic alertness demonstrated increased mean test scores for the post-training condition; however, only the reaction time change was marginally significant, and this finding was only significant with a small effect size. The post- and pre-follow-up evaluations indicated no significant changes in any of these parameters (Tables 3-5).

Table 3 Attentional performance before and after NF training

\begin{tabular}{|c|c|c|c|c|c|c|c|c|c|c|c|}
\hline Parameter & N (T0) & $\mathbf{N}(\mathrm{TI})$ & $\begin{array}{l}\text { Mean } \\
\text { score (T0) }\end{array}$ & $\begin{array}{l}\text { Mean } \\
\text { score (TI) }\end{array}$ & SD (T0) & SD (TI) & $d f$ & $t / z$ score & $P$-value & $P^{\prime}$-value & $\begin{array}{l}\text { Effect } \\
\text { sizes }\end{array}$ \\
\hline Divided Attention & 23 & 22 & & & & & & & & & \\
\hline RT auditive & 23 & 22 & 799.67 & 713.38 & 164.17 & 106.70 & 20 & 3.71 & 0.0014 & 0.0084 & 0.62 \\
\hline RT visual & 23 & 22 & $\mathrm{I}, 058.05$ & $\mathrm{I}, 045.76$ & 204.31 & 216.33 & 20 & 0.29 & 0.77 & 0.54 & 0.13 \\
\hline SD auditive & 23 & 22 & 249.10 & 176.62 & 136.76 & 77.48 & 20 & 2.85 & 0.010 & 0.050 & 0.60 \\
\hline SD visual & 23 & 22 & 354.90 & 326.52 & 147.05 & 136.67 & 20 & 1.07 & 0.30 & 1.19 & 0.26 \\
\hline Errors & 23 & 22 & 2.76 & 2.29 & 4.15 & 3.23 & 20 & 0.85 & 0.41 & 1.22 & 0.13 \\
\hline Misses & 23 & 22 & 5.67 & 5.24 & 3.89 & 4.39 & 20 & 0.72 & 0.48 & 0.96 & 0.09 \\
\hline Alertness & 23 & 22 & & & & & & & & & \\
\hline RT & 23 & 22 & 316.38 & 346.86 & 71.66 & 100.90 & 20 & -2.65 & 0.015 & 0.061 & 0.35 \\
\hline SD & 23 & 22 & 75.90 & 97.52 & 43.11 & 66.04 & 20 & -1.69 & 0.11 & 0.21 & 0.39 \\
\hline Misses & 23 & 22 & 0.62 & 0.62 & 1.16 & 1.56 & 20 & 0 & I & I & 0 \\
\hline Phasic alertness & 23 & 22 & 0.04 & 0.10 & 0.11 & 0.14 & 20 & -2.27 & 0.034 & 0.102 & 0.46 \\
\hline SSvis & 23 & 22 & & & & & & & & & \\
\hline Number of trials & 23 & 22 & 56.00 & 61.22 & 11.52 & 11.33 & 17 & -2.21 & $0.04 I$ & 0.124 & 0.46 \\
\hline RT mean & 23 & 22 & $1,084.04$ & $1,072.12$ & 317.17 & 294.87 & 20 & 0.17 & 0.87 & 0.78 & 0.039 \\
\hline $\mathrm{SD}$ mean & 23 & 22 & 620.83 & 582.11 & 259.54 & 248.48 & 17 & 0.65 & 0.52 & 1.05 & 0.15 \\
\hline Errors & 23 & 22 & 22.33 & 17.24 & 12.63 & 11.54 & 20 & 2.44 & 0.024 & 0.096 & 0.42 \\
\hline SAdots & 22 & 22 & & & & & & & & & \\
\hline Correct responses, mean RT & 22 & 22 & $\mathrm{I}, 378.74$ & $\mathrm{I}, 25 \mathrm{I} .95$ & 585.29 & 558.10 & 19 & 3.91 & 0.00095 & 0.0028 & 0.22 \\
\hline SD mean & 22 & 22 & 3.07 & 3.01 & 1.46 & 1.47 & 19 & 0.29 & 0.77 & 0.78 & 0.043 \\
\hline Incorrect responses (N) & 22 & 22 & 50.65 & 47.45 & 28.47 & 30.73 & 19 & 0.55 & 0.59 & 1.18 & 0.108 \\
\hline
\end{tabular}

Notes: The mean scores of the TAP subtests "Divided Attention" and "Alertness" and the results of the "SSvis" and "SAdots" subtests of the ANTs at two time points of assessment (pre-training [T0], post-training [TI]) are given. $d f=$ degrees of freedom, $P=$ significance (two-tailed), $P^{\prime}=$ significance (two-tailed) after $\alpha$-adjustment, $d=$ estimated effect size (Cohen's d), normal distribution according to Shapiro-Wilk and Kolmogorov-Smirnov tests.

Abbreviations: ANTs, Amsterdam Neuropsychological Tests; NF, neurofeedback; SAdots, Sustained Attention Dots; SSvis, Shifting Attentional Set; SD, standard deviation; TAP, Test for Attentional Performance; RT, reaction time; SD, standard deviation. 
Table 4 Attentional performance after NF training and at follow-up

\begin{tabular}{|c|c|c|c|c|c|c|c|c|c|c|c|}
\hline Parameter & $\mathbf{N}(\mathrm{TI})$ & $\mathbf{N}(\mathrm{T} 2)$ & $\begin{array}{l}\text { Mean } \\
\text { score (TI) }\end{array}$ & $\begin{array}{l}\text { Mean } \\
\text { score (T2) }\end{array}$ & SD (TI) & SD (T2) & $d f$ & t/z-score & $P$-value & $P^{\prime}$-value & $\begin{array}{l}\text { Effect } \\
\text { sizes }\end{array}$ \\
\hline Divided Attention & 22 & 14 & & & & & & & & & \\
\hline RT auditive & 22 & 14 & 753.08 & 690.62 & 118.79 & 97.65 & 12 & 1.90 & $0.08 I$ & 0.41 & 0.57 \\
\hline RT visual & 22 & 14 & I,II6.I5 & $1,041.15$ & 212.86 & 204.12 & 12 & 1.24 & 0.24 & 0.47 & 0.36 \\
\hline SD auditive & 22 & 14 & 207.31 & $\mathrm{I}, 67.77$ & 99.25 & 61.90 & 12 & 1.66 & 0.12 & 0.37 & 0.48 \\
\hline SD visual & 22 & 14 & 363.15 & 330.62 & 135.28 & $|65.4|$ & 12 & 0.72 & 0.48 & 0.48 & 0.22 \\
\hline Errors & 22 & 14 & 2.85 & 1.69 & 3.67 & 1.89 & 12 & 1.702 & 0.11 & 0.46 & 0.395 \\
\hline Misses & 22 & 14 & 5.92 & 4.00 & 4.39 & 3.21 & 12 & 2.35 & 0.037 & 0.22 & $0.500 \mathrm{I}$ \\
\hline Alertness & 22 & 14 & & & & & & & & & \\
\hline $\mathrm{RT}$ & 22 & 14 & 372.31 & 309.31 & 103.62 & 58.17 & 12 & 2.42 & 0.033 & 0.132 & 0.75 \\
\hline SD & 22 & 14 & 106.31 & 74.00 & 66.24 & 32.50 & 12 & 1.75 & 0.11 & 0.21 & 0.62 \\
\hline Misses & 22 & 14 & 0.85 & 0.00 & 1.95 & 0.00 & 12 & 1.56 & 0.14 & 0.14 & 0.61 \\
\hline Phasic alertness & 22 & 14 & 0.10 & 0.042 & 0.14 & 0.105 & 12 & 2.21 & 0.048 & 0.144 & 0.47 \\
\hline SSvis & 22 & 13 & & & & & & & & & \\
\hline Number of trials & 22 & 13 & 60.90 & 69.00 & 12.57 & 7.77 & 9 & -2.82 & 0.020 & 0.040 & 0.77 \\
\hline RT mean & 22 & 13 & $\mathrm{I}, \mathrm{I} 33.37$ & 930.63 & 335.69 & 253.10 & 12 & 3.54 & 0.004 & 0.012 & 0.68 \\
\hline SD mean & 22 & 13 & 539.53 & 439.60 & 197.17 & 157.84 & 9 & $\mathrm{I} .80 \mathrm{I}$ & 0.11 & 0.11 & 0.56 \\
\hline Errors & 22 & 13 & 17.62 & 9.30 & $1 \mathrm{l} .44$ & 7.48 & 12 & 3.68 & 0.003 & 0.012 & 0.86 \\
\hline SAdots & 22 & 13 & & & & & & & & & \\
\hline Correct responses, mean RT & 22 & 13 & I,384.82 & $1,238.54$ & 586.79 & $5 \mid 5.31$ & 12 & 3.197 & 0.008 & 0.0240 & 0.26 \\
\hline SD mean & 22 & 13 & 3.29 & 3.12 & 1.14 & 1.57 & 12 & 0.55 & 0.59 & 0.59 & 0.13 \\
\hline Incorrect responses $(\mathrm{N})$ & 22 & 13 & 53.00 & 48.54 & 30.59 & 36.75 & 12 & 0.76 & 0.46 & 0.92 & 0.13 \\
\hline
\end{tabular}

Notes: The mean scores of the TAP subtests "Divided Attention" and "Alertness" and the results of the "SSvis" and "SAdots" subtests of the ANTs at two time points of assessment (post-training [TI], 6-month follow-up [T2]) are given. $d f=$ degrees of freedom, $P=$ significance (two-tailed), $P^{\prime}=$ significance (two-tailed) after $\alpha$-adjustment, $d=$ estimated effect size (Cohen's $d$ ), normal distribution according to Shapiro-Wilk and Kolmogorov-Smirnov tests.

Abbreviations: ANTs, Amsterdam Neuropsychological Tests; NF, neurofeedback; SAdots, Sustained Attention Dots; SSvis, Shifting Attentional Set; SD, standard deviation;

TAP, Test for Attentional Performance; RT, reaction time; SD, standard deviation.

Table 5 Attentional performance before NF training and at follow-up

\begin{tabular}{|c|c|c|c|c|c|c|c|c|c|c|c|}
\hline Parameter & N (T0) & N (T2) & $\begin{array}{l}\text { Mean } \\
\text { score (T0) }\end{array}$ & $\begin{array}{l}\text { Mean } \\
\text { score (T2) }\end{array}$ & SD (T0) & SD (T2) & $d f$ & t/z-score & $P$-value & $P^{\prime}$-value & $\begin{array}{l}\text { Effect } \\
\text { sizes }\end{array}$ \\
\hline Divided Attention & 23 & 14 & & & & & & & & & \\
\hline RT auditive & 23 & 14 & 817.62 & 674.69 & 161.23 & 105.98 & 12 & 4.10 & 0.001 & 0.01 & 1.05 \\
\hline RT visual & 23 & 14 & $|, 0| 3.3 \mid$ & 982.62 & $|50.4|$ & 217.18 & 12 & 0.78 & 0.45 & 0.54 & 0.16 \\
\hline SD auditive & 23 & 14 & 293.62 & 162.69 & 144.96 & 65.04 & 12 & 4.02 & 0.00 & 0.01 & 1.17 \\
\hline SD visual & 23 & 14 & 336.23 & 290.85 & $|4| .5 \mid$ & $|46.4|$ & 12 & 1.06 & 0.31 & 0.62 & 0.32 \\
\hline Errors & 23 & 14 & 3.62 & 1.54 & 4.91 & 1.94 & 12 & 2.02 & 0.07 & 0.20 & 0.556 \\
\hline Misses & 23 & 14 & 6.00 & 3.77 & 3.87 & 3.24 & 12 & 2.12 & 0.055 & 0.22 & 0.6244 \\
\hline Alertness & 23 & 14 & & & & & & & & & \\
\hline RT & 23 & 14 & 330.23 & 298.54 & 85.14 & 62.23 & 12 & 1.89 & 0.083 & 0.330 & 0.43 \\
\hline SD & 23 & 14 & 77.77 & 69.46 & 47.19 & 33.87 & 12 & 0.93 & 0.37 & 0.74 & 0.20 \\
\hline Misses & 23 & 14 & 0.62 & 0.00 & 1.19 & 0.00 & 12 & 1.86 & 0.09 & 1.00 & 0.73 \\
\hline Phasic alertness & 23 & 14 & 0.04 & 0.04 & 0.13 & 0.10 & 12 & 0.16 & 0.874 & 2.621 & 0.03 \\
\hline SSvis & 23 & 13 & & & & & & & & & \\
\hline Number of trials & 23 & 13 & 59.00 & 68.78 & 11.63 & 8.21 & 8 & -2.21 & 0.027 & 0.082 & 0.97 \\
\hline RT mean & 23 & 13 & $1,066.46$ & 928.89 & $312.8 \mid$ & 264.27 & 11 & 1.83 & 0.094 & 0.188 & 0.48 \\
\hline SD mean & 23 & 13 & 539.58 & 442.94 & 201.16 & 167.04 & 8 & 1.78 & 0.11 & 0.11 & 0.52 \\
\hline Errors & 23 & 13 & 21.58 & 9.33 & 11.85 & 7.81 & II & 3.56 & 0.004 & 0.018 & 1.22 \\
\hline SAdots & 22 & 13 & & & & & & & & & \\
\hline Correct responses, mean RT & 22 & 13 & $1,516.69$ & $\mathrm{I}, 237.94$ & 638.16 & 538.22 & II & 4.376 & 0.001 & 0.0033 & 0.47 \\
\hline SD mean & 22 & 13 & 3.29 & 2.99 & 1.51 & 1.57 & 11 & 1.91 & 0.08 & 0.17 & 0.19 \\
\hline Incorrect responses $(\mathrm{N})$ & 22 & 13 & 54.92 & 48.00 & 31.55 & 38.33 & II & 0.95 & 0.36 & 0.36 & 0.20 \\
\hline
\end{tabular}

Notes: The mean scores of the TAP subtests "Divided Attention" and "Alertness" and the results of the "SSvis" and "SAdots" subtests of the ANTs at two time points of assessment (pre-training [T0], 6-month follow-up [T2]) are given. $d f=$ degrees of freedom, $P=$ significance (two-tailed), $P^{\prime}=$ significance (two-tailed) after $\alpha$-adjustment, $d=$ estimated effect size (Cohen's d), normal distribution according to Shapiro-Wilk and Kolmogorov-Smirnov tests.

Abbreviations: ANTs, Amsterdam Neuropsychological Tests; NF, neurofeedback; SAdots, Sustained Attention Dots; SSvis, Shifting Attentional Set; SD, standard deviation; TAP, Test for Attentional Performance; RT, reaction time; SD, standard deviation. 


\section{Divided Attention}

The mean auditive reaction time decreased from pre- to post-training conditions, as well as the mean auditive SD. The mean and SD of the visual performance, as well as the numbers of errors and misses, did not exhibit significant changes. The estimated medium effect sizes were calculated for both significant contrasts. The post-follow-up comparisons identified a trend toward faster auditive reaction times in the follow-up data; however, this trend did not survive $\alpha$-adjustment. The comparison of pre- and follow-up data demonstrated a significant reduction in the auditive reaction time from the pre- to follow-up training conditions, as well as a decrease in the mean auditive SD; both findings had large effect sizes (complete results are given in Tables 3-5). The significant results are provided in Figures 4 and 5.

\section{Shifting Attentional Set}

The mean number of trials increased from the pre- to post-training conditions, but this trend did not survive $\alpha$-adjustment. The post- to follow-up assessment demonstrated a significant increase in the mean number of trials, which indicates an improvement in performance. The prepost comparison also demonstrated a trend toward a reduction in the SD, but this trend was not statistically significant. The number of errors was decreased after NF training, but this effect did not reach statistical significance. There was a significant decrease in errors from $\mathrm{T} 1$ to $\mathrm{T} 2$ and $\mathrm{T} 0$ to $\mathrm{T} 2$, and both findings had rather large estimated effect sizes. There was no significant difference regarding the mean reaction times between T0 and T1; however, a significantly faster

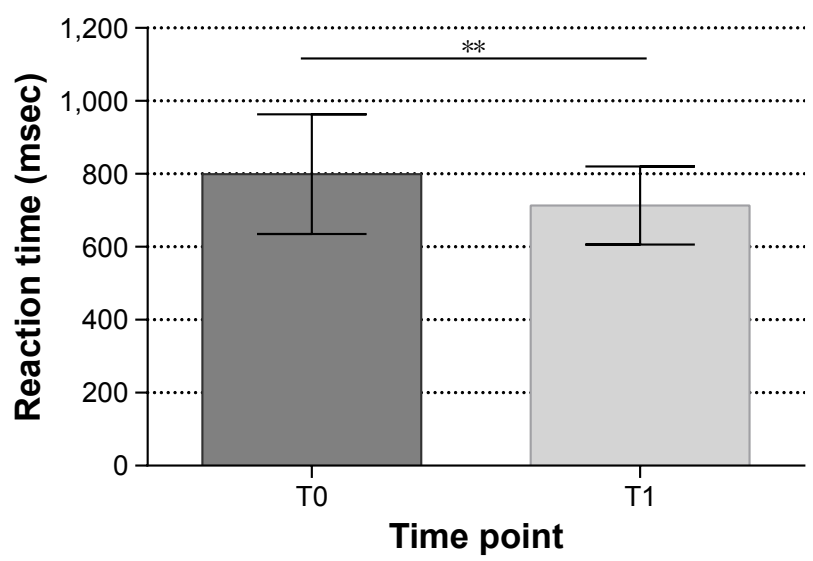

Figure 4 Mean auditive reaction time of the Divided Attention task before and after NF training.

Notes: The mean auditive reaction times (msec) at the pre- (T0) and post-training $(\mathrm{TI})$ assessment points are shown. Data are represented as mean $\pm \mathrm{SD}$. Asterisk symbol indicate the significance $\left(P^{\prime}\right)$. *** $P^{\prime} \leq 0.01$.

Abbreviations: NF, neurofeedback; SD, standard deviation.

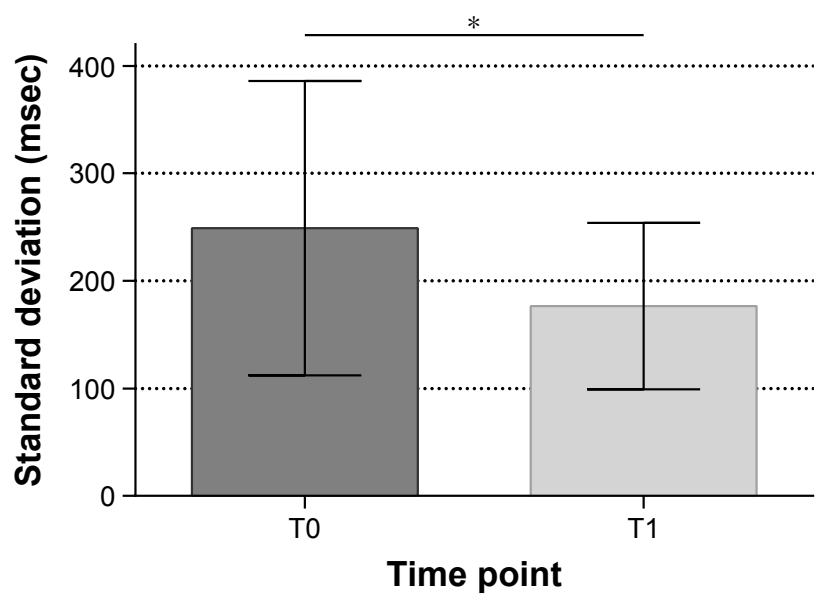

Figure 5 Mean SD of the auditive reaction time of the Divided Attention task before and after NF training.

Notes: The mean SDs $(\mathrm{msec})$ of the mean auditive reaction times at the pre(T0) and post-training (TI) assessment points are shown. Data are represented as mean \pm SD. Asterisk symbol indicate the significance $\left(P^{\prime}\right) . * P^{\prime} \leq 0.05$.

Abbreviations: NF, neurofeedback; SD, standard deviation.

reaction time was recorded for the follow-up assessment time point compared with the post-results, and a medium effect size was calculated (Tables 3-5). The significant results are shown in Figures 6 and 7.

\section{Sustained Attention Dots}

The mean reaction time of the correct responses was significantly decreased after the NF training program, but with rather small effect sizes. This decrease persisted from post to follow-up assessments, and was significant with a small effect size. A reaction time reduction in correct responses between the pre- and follow-up conditions was also significant with a small effect size. There were no significant differences in

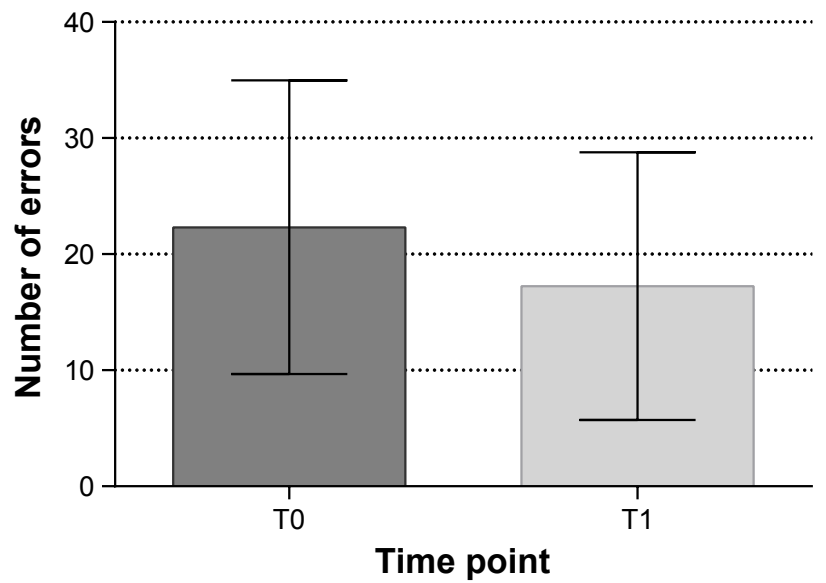

Figure 6 Number of errors in the SSvis task before and after NF training. Notes: The number of errors at the pre-training (T0) and post-training (TI) assessment points is shown. Data are represented as mean \pm SD.

Abbreviations: NF, neurofeedback; SSvis, Shifting Attentional Set; SD, standard deviation. 


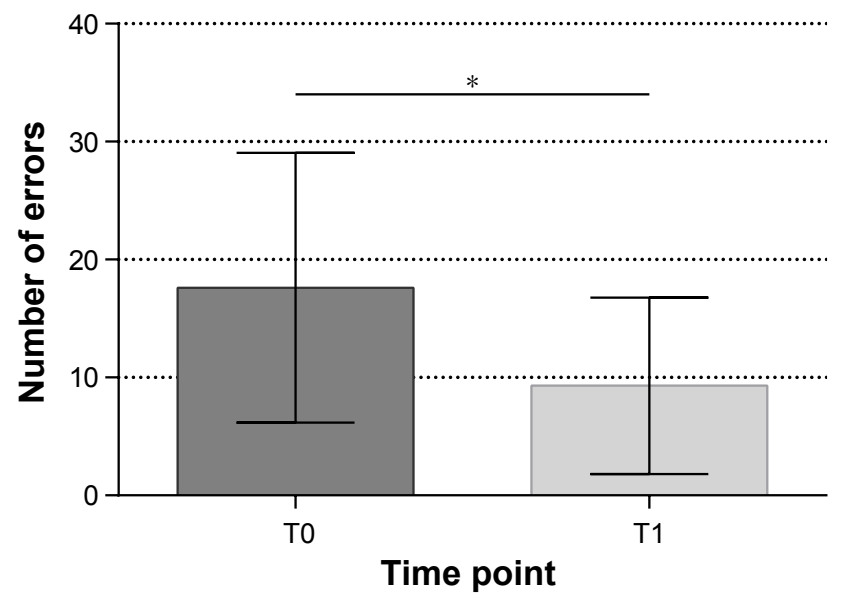

Figure 7 Number of errors in the SSvis task after NF training and at the 6-month follow-up.

Notes: The number of errors at the pre-training (T0) and post-training (TI) assessment points is shown. Data are represented as mean \pm SD. Asterisk symbol indicate the significance $\left(P^{\prime}\right)$. $* P^{\prime} \leq 0.05$.

Abbreviations: NF, neurofeedback; SSvis, Shifting Attentional Set; SD, standard deviation.

the number of errors or the SDs regarding the three time points of assessment. The complete results are summarized in Tables 3-5, and significant results are shown in Figures 8 and 9.

\section{Symptom ratings}

Although the mean scores of the inattention scale of parent ratings in the FBB-HKS-questionnaire decreased from $\mathrm{T} 0$ to $\mathrm{T} 1$, the $t$-test demonstrated no significant difference between the two time points of assessment. The teachers also reported a reduction in the mean inattention scores from $\mathrm{T} 0$ to $\mathrm{T} 1$, but

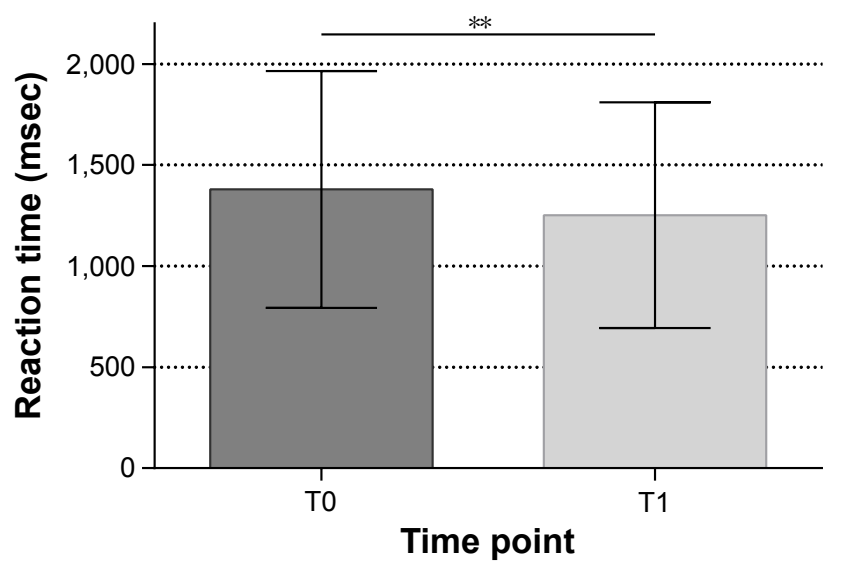

Figure 8 Mean reaction time of the SAdots task before and after NF training. Notes: The mean reaction times (msec) at the pre- (T0) and post-training (TI) assessment points are shown. Data are represented as mean $\pm \mathrm{SD}$. Asterisk symbols indicate the significance $\left(P^{\prime}\right)$. $* * P^{\prime} \leq 0.01$.

Abbreviations: NF, neurofeedback; SAdots, Sustained Attention Dots; SD, standard deviation.

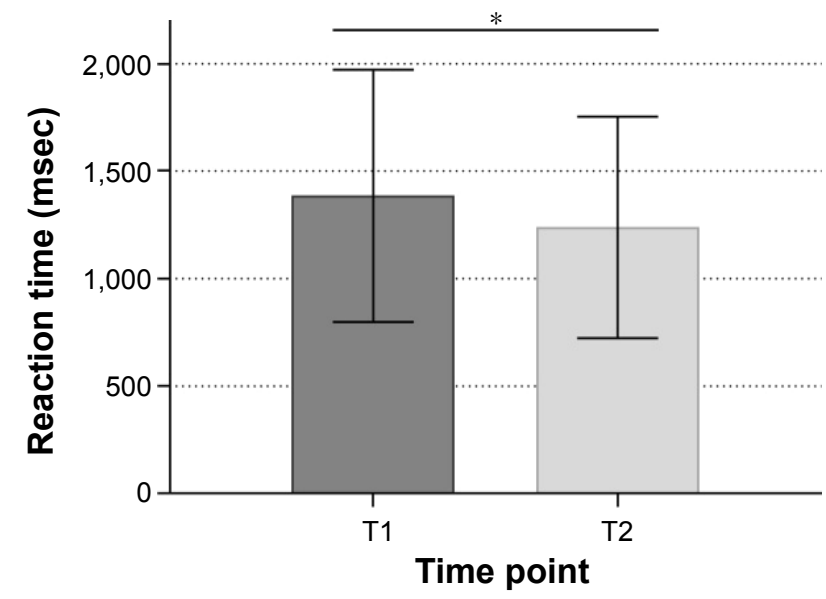

Figure 9 Mean reaction time of the SAdots task after NF training and at the 6-month follow-up.

Notes: The mean reaction times (msec) at the post-training (TI) and follow-up (T2) assessment points are shown. Data are represented as mean \pm SD. Asterisk symbols indicate the significance $\left(P^{\prime}\right)$. $* P^{\prime} \leq 0.05$.

Abbreviations: NF, neurofeedback; SAdots, Sustained Attention Dots; SD, standard deviation.

these differences were not significant. The mean $t$-scores for the CBCL's attention scale decreased from $\mathrm{T} 0$ to $\mathrm{T} 1$, but this change was not significant (Tables 6-8).

\section{SCP regulation}

As shown in Figure 3, data of all trainees on successful regulation of SCPs for the feedback, as well as transfer trials, were analyzed. Participants started with a mean percentage of correctly regulated feedback trials for negativation of $16.46 \%$, continuously increasing the percentage of correct regulation up to $25.99 \%$ at the end of phase I. At the beginning of phase II, a clear decrease in SCP regulation ability is visible, but the percentage of correct SCP correlation did rise again, and the highest percentage of correct negative regulation was reached at session $16(26.64 \%)$ and was at $24.29 \%$ at the end of phase II. For transfer trials, the mean percentage of successful regulated negativation trials started at $\sim 18.25 \%$ in session 7 and was highest at session 17 with $24.49 \%$ correctly regulated trials. A clear decrease in SCP regulation ability could also be seen in the transfer trials (20.94\% of correctly regulated negativation transfer trials in session 10 versus $15.80 \%$ of correctly regulated negativation transfer trials in session 11).

\section{Discussion}

Here, the data regarding the pre-post and follow-up comparisons of the effects of a structured SCP-based NF training program and different aspects of attentional performance in a sample of young patients with ADHD were analyzed. 
Table 6 Symptom ratings before and after NF training

\begin{tabular}{|c|c|c|c|c|c|c|c|c|c|c|c|}
\hline Parameter & N (T0) & $\mathbf{N}(\mathrm{TI})$ & $\begin{array}{l}\text { Mean } \\
\text { score (T0) }\end{array}$ & $\begin{array}{l}\text { Mean } \\
\text { score (TI) }\end{array}$ & SD (T0) & SD (TI) & $d f$ & t-score & $P$-value & $P^{\prime}$-value & $\begin{array}{l}\text { Effect } \\
\text { sizes }\end{array}$ \\
\hline \multicolumn{12}{|l|}{ Parent ratings } \\
\hline FBB-HKS (DSM-4) & 20 & 18 & & & & & & & & & \\
\hline Inattention & 20 & 18 & 1.59 & 1.45 & 0.67 & 0.66 & 16 & 0.98 & 0.34 & 1.02 & 0.22 \\
\hline $\mathrm{CBCL} / 4-18$ & 22 & 20 & & & & & & & & & \\
\hline Attention problems & 22 & 20 & 65.58 & 64.11 & 9.75 & 8.83 & 18 & 0.94 & 0.36 & 0.72 & 0.16 \\
\hline \multicolumn{12}{|l|}{ Teacher ratings } \\
\hline \multicolumn{12}{|l|}{ FBB-HKS (DSM-4) } \\
\hline Inattention & 17 & 14 & 1.80 & 1.68 & 0.70 & 0.69 & 10 & 0.86 & $0.4 I$ & $0.4 I$ & 0.17 \\
\hline
\end{tabular}

Notes: The mean scores of the FBB-HKS symptom ratings/checklist, severity scores for inattention and the CBCL/4-I8 at the two time points of assessment (pre-training [TO] and post [TI]) are given. $d f=$ degrees of freedom, $P=$ significance (two-tailed), $P^{\prime}=$ significance (two-tailed) after $\alpha$-adjustment, $d=$ estimated effect size (Cohen's $d$ ), normal distribution according to Shapiro-Wilk and Kolmogorov-Smirnov tests.

Abbreviations: CBCL/4-18, Child Behavior Checklist/4-I8; DSM, Diagnostic and Statistical Manual of Mental Disorders; NF, neurofeedback; SD, standard deviation; FBB-HKS, Fremdbeurteilungsbogen für Hyperkinetische Störungen.

Overall, SCP-based NF had positive effects on attentional performance, such as decreases in the reaction times between the pre- and post-assessment points in the divided and sustained attention tasks. These changes were somewhat stable over time as they remained visible in the 6-month follow-up assessment. For the Divided Attention task, a reduction in the SD of the reaction time (auditive) was detected after NF training, which might be interpreted as a more constant attentional performance. Furthermore, the participants did not make additional errors while increasing their work pace; if anything, they reduced the number of mistakes in the pre-/follow-up and post-/follow-up comparisons in the set-shifting task.

Overall, the observed effects from the current analysis can be summarized in terms of an improvement to be detected in different reaction time values in various tests administered, and these effects were found to be of small-to-medium magnitude. For example, there was a decrease in the mean auditive reaction time in the Divided Attention task (T0-T1, medium effect sizes), and this effect was persistent over time (T0-T2 comparison, larger effect sizes). The mean reaction time in the SAdots task was faster from T0 to T1 and T1 to T2 (small effect sizes). In the SSvis, the mean reaction time was significantly reduced from T1 to T2 (medium effect sizes), and patients with ADHD also demonstrated faster reaction times in the SAdots task (correct responses) after NF training (which also persisted to T2) as well as in the Alertness task (small effect size). As regards other test parameters assessed in the current approach, the patients were also able to increase the number of trials from $\mathrm{T} 1$ to $\mathrm{T} 2$ and significantly reduce the number of errors from T1 to T2 and T0 to T2 (SSvis, large effect sizes). There were no significant changes in the number of errors (SAdots task).

Overall, the effects found on parameters related to different reaction times are consistent with previous research involving adult patients with $\mathrm{ADHD},{ }^{41}$ in which effects of SCP-related NF training were found for reaction times as well as reaction time variability, and there was also a tendency for an increased CNV. Because the CNV as an event-related potential is believed to reflect the underlying resource allocation of neural circuits during cognitive preparation, the current preliminary findings map on to existing knowledge on

Table 7 Symptom ratings after NF training and at the 6-month follow-up

\begin{tabular}{|c|c|c|c|c|c|c|c|c|c|c|c|}
\hline Parameter & $\mathbf{N}(\mathrm{TI})$ & $\mathbf{N}(\mathrm{T} 2)$ & $\begin{array}{l}\text { Mean } \\
\text { score (TI) }\end{array}$ & $\begin{array}{l}\text { Mean } \\
\text { score (T2) }\end{array}$ & SD (TI) & SD (T2) & $d f$ & t-score & $P$-value & $P^{\prime}$-value & $\begin{array}{l}\text { Effect } \\
\text { sizes }\end{array}$ \\
\hline \multicolumn{12}{|l|}{ Parent ratings } \\
\hline FBB-HKS (DSM-4) & 18 & 14 & & & & & & & & & \\
\hline Inattention & 18 & 14 & 1.40 & 1.64 & 0.70 & 0.63 & II & -1.28 & 0.23 & 0.68 & 0.37 \\
\hline $\mathrm{CBCL} / 4-18$ & 20 & 14 & & & & & & & & & \\
\hline Attention problems & 20 & 14 & 65.43 & 63.00 & 8.74 & 7.83 & 13 & 1.32 & 0.21 & 0.21 & 0.29 \\
\hline \multicolumn{12}{|l|}{ Teacher ratings } \\
\hline Inattention & 14 & 9 & 1.60 & 1.25 & $0.7 \mid$ & 0.72 & 7 & 1.32 & 0.23 & 0.46 & 0.49 \\
\hline
\end{tabular}

Notes: The mean scores of the FBB-HKS symptom ratings/checklist, severity scores for inattention and the CBCL/4-I8 at the two time points of assessment (post [TI] and follow-up [T2]) are given. $d f=$ degrees of freedom, $P=$ significance (two-tailed), $P^{\prime}=$ significance (two-tailed) after $\alpha$-adjustment, $d=$ estimated effect size (Cohen's $d$ ), normal distribution according to Shapiro-Wilk and Kolmogorov-Smirnov tests.

Abbreviations: CBCL/4-18, Child Behavior Checklist/4-18; DSM, Diagnostic and Statistical Manual of Mental Disorders; NF, neurofeedback; SD, standard deviation; FBB-HKS, Fremdbeurteilungsbogen für Hyperkinetische Störungen. 
Table 8 Symptom ratings before NF training and at the 6-month follow-up

\begin{tabular}{|c|c|c|c|c|c|c|c|c|c|c|c|}
\hline Parameter & $\mathbf{N}(\mathrm{T} 0)$ & $\mathbf{N}(\mathrm{T} 2)$ & $\begin{array}{l}\text { Mean score } \\
\text { (T0) }\end{array}$ & $\begin{array}{l}\text { Mean score } \\
\text { (T2) }\end{array}$ & SD (T0) & SD (T2) & $d f$ & t-score & $P$-value & $P^{\prime}$-value & $\begin{array}{l}\text { Effect } \\
\text { sizes }\end{array}$ \\
\hline \multicolumn{12}{|l|}{ Parent ratings } \\
\hline FBB-HKS (DSM-4) & 20 & 14 & & & & & & & & & \\
\hline Inattention & 20 & 14 & 1.71 & 1.62 & 0.57 & 0.61 & 12 & 0.49 & 0.63 & 0.63 & 0.95 \\
\hline $\mathrm{CBCL} / 4-18$ & 22 & 14 & & & & & & & & & \\
\hline Attention problems & 22 & 14 & 66.64 & 63.00 & 10.05 & 7.83 & 13 & 2.08 & 0.06 & 0.12 & 0.40 \\
\hline \multicolumn{12}{|l|}{ Teacher ratings } \\
\hline Inattention & 17 & 9 & 1.97 & 1.21 & 0.91 & 0.76 & 6 & 2.57 & 0.04 & 0.13 & 0.90 \\
\hline
\end{tabular}

the correlates of successful NF training regulation in young people with ADHD. ${ }^{42}$ In particular, because a more general slowing in EEG-related brain activity and also a decline in event-related potential amplitudes such as the CNV can be detected in patients with ADHD of different age groups, ${ }^{43}$ the current findings are in-line with previous research suggesting a cortical hypoarousal in patients with ADHD. This cortical hypoarousal can be related to changes in reaction time, which can be clinically assessed through neuropsychological test batteries. More recent findings showed that children with ADHD presented with delayed reaction times, poorer behavioral performance, larger cue-elicited $\mathrm{P} 2$ and reduced CNV in the preparation stage, and that a larger CNV amplitude predicted better task performance. ${ }^{44}$

The delayed effects of SCP-based NF on error presentation and reaction time performance in the shifting attention task as well as the observed changes after need to be interpreted with a high degree of caution. Because no NF-related intervention was administered between $\mathrm{T} 1$ and $\mathrm{T} 2$ in the current approach, the observed improvements between $\mathrm{T} 1$ and $\mathrm{T} 2$ are likely to be due to other interventions or improvements related to the patients' situation and thus cannot be directly attributed to the NF intervention. It is possible that these factors do, to some extent, have an influence on the improvements between T0 und T1 as well. If future research could detect further evidence for secondary, prolonged treatment effects and/or delayed improvements in neuropsychological parameters (eg, set shifting) and these improvements could be attributed to the clinical use of SCP-based NF in young patients with ADHD, the current findings could be taken into account. However, at the current stage, immediate and directly related effects of the SCP training administered in the current approach cannot be concluded, and future research on the prolonged effects as well as possible delayed but training-related improvements is necessary, which is why the current data on set shifting are presented on a descriptive level.

The rationale for an initially higher number of NF training sessions in the first 2 weeks of the training (which was conducted during 2-week school holidays) was that a short interval between the first/initial training sessions would allow the patients to practice more frequently, to improve their ability to regulate ADHD symptom-related brain activity and to familiarize themselves quickly with the training setup and explore their own individual strategies. In addition, allowing familiarization with the NF setup during school holidays without a possible pressure in terms of not having enough time for school-related work/homework and other commitments hopefully enabled patients to engage with the treatment team. As shown in Tables 9 and 10, participants also improved their regulation of negative SCPs during the intensive training phase I. The second training phase (with an equal number of total sessions as phase I but with fewer sessions per week, thus a longer time period) was used to allow feasibility in terms of logistics and the ability to schedule regular appointments during the school term and to serve as a more continuous training phase. Overall, the use of such a setup was well perceived by the patients and their families, which is an important clinical aspect when it comes to having the patients and their families and carers engage with a service providing treatment. The SCP regulation abilities shown at the end of phase I were reduced at the beginning of phase II and increased again as phase II went on. Regular training on all weekdays in phase II might have increased the participant's ability to create SCP changes and might also have led to greater improvements regarding attention. The patient's ability to create higher SCP changes in positivity trials at the beginning of phase I might be associated with ADHD symptoms such as inattention. At the end of session I, 
Table 9 Regulation during feedback trials

\begin{tabular}{|c|c|c|c|c|c|}
\hline \multirow[t]{2}{*}{ Session } & \multirow[t]{2}{*}{$\mathbf{N}$} & \multicolumn{2}{|c|}{$\begin{array}{l}\text { Feedback } \\
\text { negativation }\end{array}$} & \multicolumn{2}{|l|}{$\begin{array}{l}\text { Feedback } \\
\text { positivation }\end{array}$} \\
\hline & & $\begin{array}{l}\text { Mean \% of } \\
\text { correctly } \\
\text { regulated } \\
\text { trials }\end{array}$ & SD & $\begin{array}{l}\text { Mean \% of } \\
\text { correctly } \\
\text { regulated } \\
\text { trials }\end{array}$ & SD \\
\hline Session I & 23 & 16.46 & $13.5 \mid$ & 19.70 & 16.88 \\
\hline Session 2 & 24 & 14.83 & 11.62 & 22.86 & 16.48 \\
\hline Session 3 & 24 & 15.90 & 14.16 & 17.75 & 11.43 \\
\hline Session 4 & 24 & $|7.7|$ & $|5.4|$ & 22.13 & 13.39 \\
\hline Session 5 & 24 & 16.75 & 12.47 & 19.74 & 12.65 \\
\hline Session 6 & 24 & 22.33 & 16.47 & 22.82 & 14.50 \\
\hline Session 7 & 24 & 20.38 & 15.60 & 23.39 & 17.10 \\
\hline Session 8 & 24 & 18.72 & 16.24 & 23.39 & 19.33 \\
\hline Session 9 & 23 & 22.67 & 16.42 & 22.93 & 21.59 \\
\hline Session 10 & 24 & 25.99 & 21.40 & 18.42 & 10.17 \\
\hline Session II & 24 & 15.52 & 13.99 & 28.60 & 20.67 \\
\hline Session 12 & 24 & 23.76 & 23.00 & 24.03 & 21.18 \\
\hline Session 13 & 24 & 17.67 & 17.49 & 25.81 & 22.38 \\
\hline Session 14 & 23 & 21.11 & 19.71 & 23.03 & 26.14 \\
\hline Session 15 & 23 & 21.71 & 14.77 & 26.42 & 23.67 \\
\hline Session 16 & 23 & 26.64 & 18.62 & 21.67 & 24.30 \\
\hline Session 17 & 23 & 23.27 & 20.26 & 32.05 & 24.60 \\
\hline Session 18 & 22 & 22.39 & 19.89 & 23.17 & 20.83 \\
\hline Session 19 & 21 & 17.40 & 12.63 & 25.86 & 22.97 \\
\hline Session 20 & 21 & 24.29 & 20.53 & 25.50 & 21.97 \\
\hline
\end{tabular}

Note: The mean percentage (\%) of correctly regulated feedback trials for positivation and negativation is given.

Abbreviation: SD, standard deviation.

Table 10 Regulation during transfer trials

\begin{tabular}{|c|c|c|c|c|c|}
\hline \multirow[t]{2}{*}{ Session } & \multirow[t]{2}{*}{$\mathbf{N}$} & \multicolumn{2}{|c|}{$\begin{array}{l}\text { Transfer } \\
\text { negativation }\end{array}$} & \multicolumn{2}{|l|}{$\begin{array}{l}\text { Transfer } \\
\text { positivation }\end{array}$} \\
\hline & & $\begin{array}{l}\text { Mean \% of } \\
\text { correctly } \\
\text { regulated } \\
\text { trials }\end{array}$ & SD & $\begin{array}{l}\text { Mean \% of } \\
\text { correctly } \\
\text { regulated } \\
\text { trials }\end{array}$ & SD \\
\hline Session I & 23 & & & & \\
\hline Session 2 & 24 & & & & \\
\hline Session 3 & 24 & & & & \\
\hline Session 4 & 24 & & & & \\
\hline Session 5 & 24 & & & & \\
\hline Session 6 & 24 & & & & \\
\hline Session 7 & 24 & 18.25 & 17.94 & 24.60 & 21.01 \\
\hline Session 8 & 24 & 20.25 & 16.40 & 19.26 & 17.20 \\
\hline Session 9 & 23 & 24.38 & 20.67 & 22.10 & 20.63 \\
\hline Session 10 & 24 & 20.94 & 19.77 & 18.74 & $|3.5|$ \\
\hline Session II & 24 & 15.80 & 16.67 & 28.19 & 14.69 \\
\hline Session 12 & 24 & 16.86 & 20.51 & 20.60 & 17.11 \\
\hline Session 13 & 24 & 18.03 & 17.60 & 23.85 & 17.81 \\
\hline Session 14 & 23 & 21.42 & 17.57 & 18.36 & 20.52 \\
\hline Session 15 & 23 & 24.12 & 15.82 & 22.96 & 16.54 \\
\hline Session 16 & 23 & 24.09 & 16.15 & 18.83 & 15.20 \\
\hline Session 17 & 23 & 24.49 & 22.51 & 26.67 & 17.35 \\
\hline Session 18 & 22 & 21.36 & 16.39 & 23.80 & 24.11 \\
\hline Session 19 & 21 & 17.46 & 11.19 & 19.27 & 16.36 \\
\hline Session 20 & 21 & 23.36 & 16.34 & 21.05 & 16.98 \\
\hline
\end{tabular}

Note: The mean percentage (\%) of correctly regulated transfer feedback trials for positivation and negativation is given.

Abbreviation: SD, standard deviation. the patient's ability to regulate negative SCPs is higher than the regulation of positive SCPs for transfer trials, but not for feedback trials.

As regards further key implications of the used approach, these particular aspects relate to the scheduling and the intensity of the training administered, and to the clinical feasibility. One important aspect relates to the fact that patients and their families and carers highly appreciated and tolerated the used approach (two-phase design, with phase I running during the school holidays), which has important implications with regard to the feasibility of such training programs and limited available time. Moreover, despite a rather high training intensity within phase I of the program (daily trainings on weekdays), none of the patients had to drop out because of side effects (eg, headaches), which supports the clinical feasibility of the used approach in principle. The data of the current analysis are also of particular value for future research as they allow the calculation of adequate sample sizes for prospective studies, especially when it comes to using NF of SCPs in young patients with ADHD within a two-phased approach involving an initial intensive training phase (five sessions per week on workdays during phase I) and two sessions per week on workdays during phase II. For instance, to detect a significant difference in means in one group of patients (eg, before and after training) in the auditive reaction time in the Divided Attention task with an effect size of 0.62 (as found in the current analysis), an alpha level of 0.05 and with a power of 0.958 , a total analyzed sample size of at least $\mathrm{N}=18$ subjects, would be necessary (repeatedmeasures ANOVA, within-factors, assuming a correlation of 0.1 between repeated-measures variables, nonsphericity correction $=1$; power calculation/estimation was performed with G*Power Software (Faul et al, 2007, 2009). ${ }^{45,46}$

The current data are subject to a number of limitations. First, the retrospective analysis approach and the absence of a control group must be considered, which was not included because this study focuses on the clinical data of a structured NF training program and not a randomized controlled trial. Moreover, no placebo condition was used because having a patient participation in 20 sessions of a placebo feedback would also raise ethical questions. The neurocognitive tasks that were used in the current training project were designed to be used for multiple assessments in the same patient and have adequate test/retest reliability. However, practice effects cannot be ruled out completely, which applies to any study aiming to assess changes in performance over time in the same subjects. No significant statistical effects were identified in the results of the questionnaire data (behavioral/symptom 
ratings); however, the questionnaires were not always returned. Because the parents and children actively decided to participate in the NF training (some parents even paid for the treatment), they might be biased in the judgment of the child's behavior. Because the questionnaires for symptom ratings were distributed at the three time points of assessment, with a period of $\sim 7-8$ months between the pre- and follow-up assessments, the participants sometimes had different teachers or even switched schools; thus, the individuals who filled out the first symptom ratings were sometimes not the individuals who completed the two subsequent ratings. Furthermore, participants were not excluded when the questionnaires were not returned or a training session was missed because of illness or school activities because this NF program was a pilot project; it examined the use of a new algorithm for NF-based treatment and was not a randomized controlled clinical study. Finally, it is often a more general criticism that NF training requires a very close interaction between the patient and coach and that unspecific effects, for example, the positive feedback of the trainer or reminders to sit still, might lead to a positive effect on attention and not the NF itself. ${ }^{41}$ These factors cannot be ruled out when the data are obtained from real clinical settings and not a trial-specific environment. It is also possible that the first suggested strategies used for creating SCP changes (such as roller coaster vs dreaming) may have served the role of a placebo, thus adding to the observed improvements from T1 to T2. However, these strategies were only used as a starting point for participants, and all subjects in this program were encouraged to explore their own individual strategies from this point on. Nevertheless, despite these limitations, the data obtained in the current investigation have their clinical value. In particular, this is the first pilot data that enabled the reliable calculation of sample size estimations for future large-scale studies, in particular, because it can be assumed that previous studies that involved NF were underpowered. Moreover, the use of a rather intensive first phase of training with almost daily sessions (phase I) is new and has not been performed in previously published research. Future studies that aim to develop evidence-based training algorithms should consider a further evaluation of an intensive training phase to optimize the slope of the learning curve with regard to SCP-based cortical self-regulation.

A considerable number of studies that used NF have been completed in recent years; in particular, studies have used semi-active control conditions, which include conditions that are not expected to have an effect on ADHD symptoms, such as EMG biofeedback ${ }^{42}$ or a computerized cognitive skills training. Active control conditions compare two treatment methods that do have an effect on ADHDrelated symptoms, for example, theta/beta with $\mathrm{SCP} \mathrm{NF}^{23}$ or pharmacological treatments to NF. ${ }^{43,44}$ Placebo-controlled studies are considered to be the scientific gold standard, but they are difficult to conduct for NF. In this particular scenario, one treatment group would receive a feedback that has no correlation with brain activity, and the treatment would need to be blinded to avoid an expectancy bias by trainers and therapists. The values of operant conditioning are applied in both training groups, such as positive reinforcement for positive achievements. For the control group, an automated threshold would need to be used to ensure blinding of the trainer and the patient would not be rewarded according to achievements in self-regulation. This method does not value the principles of conditioning and learning theory. Moreover, in a real clinical setting, such as the setting used in the current approach, it would also be considered unethical to prevent the actual treatment of children with ADHD who suffer from high psychological stress in everyday life because of their condition, thereby providing them sham NF rather than comparing two valuable treatment options.

\section{Conclusion}

Several clinical advantages of the current NF program include: a structured NF training program with the first positive but preliminary results on attention in children with ADHD, even with a fewer number of sessions than are typically applied and an intensive training phase at the beginning of the program. As discussed earlier, the limitations of the preliminary data obtained include the rather small sample size, the lack of a control group and the open-label approach because of the clinical setting. Larger sample sizes, a control group and an approach that blinds the data acquisition according to the principles of operant conditioning are needed, which should be targeted by future translational research. The current preliminary data enable the calculation of sample sizes needed for these prospective research approaches.

\section{Disclosure}

FDZ was the recipient of an unrestricted award donated by the APA, the American Psychiatric Institute for Research and Education (APIRE) and AstraZeneca (Young Minds in Psychiatry Award). He has also received research support from the German Federal Ministry for Economics and Technology, the European Commission, the Princess Margaret 
Hospital Foundation, the Telethon Perth Children's Hospital Research Fund (TPCHRF), the Telethon Kids Institute Perth, the German Society for Social Pediatrics and Adolescent Medicine, the Paul and Ursula Klein Foundation, the Dr August Scheidel Foundation, the Interdisciplinary Center for Clinical Research (IZKF) fund of the University Hospital of RWTH Aachen University, and a travel stipend donated by the GlaxoSmithKline Foundation. He is the recipient of an unrestricted educational grant, travel support and speaker honoraria by Shire Pharmaceuticals, Germany. In addition, he has received support from the Raine Foundation for Medical Research (Raine Visiting Professorship) and editorial fees from Co-Action Publishing (Sweden). The Clinic for Child and Adolescent Psychiatry, Psychosomatics and Psychotherapy at RWTH Aachen University Hospital (local project lead was FDZ) also had a joint research project with the NeuroConn company (Ilmenau, Germany, manufacturer of the used TheraPrax NF devices), the Hasomed company (Magdeburg, Germany), the Helmholtz Institute for Biomedical Engineering (section/institutes for Medical Information Technology and Medical Technology, Aachen, Germany) on the development of a new NF device (not used in the current approach). After completion of the project, TJG was an employee of the NeuroCare Group (Munich, Germany), which comprises the NeuroConn company (Ilmenau, Germany) which manufactures the used TheraPrax neurofeedback devices. However, at the time the current data were obtained, TJG was only affiliated with RWTH Aachen University (Aachen, Germany) and JARA-Brain (Aachen \& Jülich, Germany). The other authors report no conflicts of interest in this work.

\section{References}

1. Schlack R, Holling H, Kurth BM, Huss M. Die Prävalenz der Aufmerksamkeitsdefizit-/Hyperaktivitätsstörung (ADHS) bei Kindern und Jugendlichen in Deutschland. Erste Ergebnisse aus dem Kinder- und Jugendgesundheitssurvey (KiGGS) [The prevalence of attention-deficit/ hyperactivity disorder (ADHD) among children and adolescents in Germany. Initial results from the German Health Interview and Examination Survey for Children and Adolescents (KiGGS)]. Bundesgesundheitsblatt Gesundheitsforschung Gesundheitsschutz. 2007;50(5-6):827-835. German.

2. Polanczyk G, Silva de Lima M, Lessa Horta B, Biederman J, Rohde LA. The worldwide prevalence of ADHD: a systematic review and metaregression analysis. Am J Psychiatry. 2007;164(6):942-948.

3. American Psychiatric Association. Diagnostic and Statistical Manual of Mental Disorders. 5th ed. Arlington, VA: American Psychiatric Association; 2013.

4. Klein RG, Mannuzza S, Olazagasti MA, et al. Clinical and functional outcome of childhood attention-deficit/hyperactivity disorder 33 years later. Arch Gen Psychiatry. 2012;69(12):1295-1303.

5. National Institute for Health and Clinical Excellence. Attention Deficit Hyperactivity Disorder Diagnosis and Management of ADHD in Children, Young People and Adults. Leicester: British Psychological Society; 2008. [NICE clinical guideline 72].
6. Subcommittee on Attention-Deficit/Hyperactivity Disorder; Steering Committee on Quality Improvement and Management; Wolraich M, et al. ADHD: clinical practice guideline for the diagnosis, evaluation, and treatment of attention-deficit/hyperactivity disorder in children and adolescents. Pediatrics. 2011;128(5):1007-1022.

7. Elliott GR, Blasey C, Rekshan W, et al. Cognitive testing to identify children with ADHD who do and do not respond to methylphenidate. J Atten Disord. Epub 2014 Aug 13.

8. Lubar JF, Shouse MN. EEG and behavioural changes in a hyperkinetic child concurrent with training of the sensorimotor rhythm (SMR): a preliminary report. Biofeedback Self Regul. 1976;3:293-306.

9. Beauregard M, Lévesque J. Functional magnetic resonance imaging investigation of the effects of neurofeedback training on the neural bases of selective attention and response inhibition in children with attentiondeficit/hyperactivity disorder. Appl Psychophysiol Biofeedback. 2006; 31(1):3-20.

10. Stokes DA, Lappin MS. Neurofeedback and biofeedback with 37 migraineurs: a clinical outcome study. Behav Brain Funct. 2010;6:9.

11. Zhuo C, Li L. The application and efficacy of combined neurofeedback therapy and imagery training in adolescents with Tourette syndrome. J Child Neurol. 2013;29(7):965-968.

12. Heinrich H, Gevensleben H, Strehl U. Annotation: neurofeedback? Train your brain to train behaviour. J Child Psychol Psychiatry. 2007; 48(1):3-16.

13. Lubar JF. Discourse on the development of EEG diagnostics and biofeedback for attention-deficit/hyperactivity disorders. Biofeedback Self Regul. 1991;16(3):201-225.

14. Hart H, Radua J, Nakao T, Mataix-Cols D, Rubia K. Meta-analysis of functional magnetic resonance imaging studies of inhibition and attention in attention-deficit/hyperactivity disorder: exploring task-specific, stimulant medication, and age effects. JAMA Psychiatry. 2013;70(2): 185-198.

15. Elbert T, Rockstroh B, Lutzenberger W, Birbaumer N. Biofeedback of slow cortical potentials. I. Electroencephalogr Clin Neurophysiol. 1980;48(3):293-301.

16. Lutzenberger W, Elbert T, Rockstroh B, Birbaumer N. The effects of self-regulation of slow cortical potentials on performance in a signal detection task. Int J Neurosci. 1979;9(3):175-183.

17. Birbaumer N. Slow cortical potentials: plasticity, operant control, and behavioral effects. Neuroscientist. 1999;5(2):74-78.

18. Rockstroh B, Elbert T, Canavan A, Lutzenberger W, Birbaumer N. Slow Brain Potentials and Behavior. 2nd ed. Baltimore: Urban \& Schwarzenberg; 1989.

19. Rockstroh B, Elbert T, Lutzenberger W, Birbaumer N. Biofeedback: Evaluation and Therapy in Children with Attentional Dysfunctions. Berlin: Springer; 1990.

20. Heinrich H, Gevensleben H, Freisleder FJ, Moll GH, Rothenberger A. Training of slow cortical potentials in attention-deficit/hyperactivity disorder: evidence for positive behavioral and neurophysiological effects. Biol Psychiatry. 2004;55(7):772-775.

21. Gevensleben H, Holl B, Albrecht B, et al. Distinct EEG effects related to neurofeedback training in children with ADHD: a randomized controlled trial. Int J Psychophysiol. 2009;74(2):149-157.

22. Gevensleben H, Holl B, Albrecht B, et al. Is neurofeedback an efficacious treatment for ADHD? A randomised controlled clinical trial. J Child Psychol Psychiatry. 2009;50(7):780-789.

23. Leins U, Goth G, Hinterberger T, Klinger C, Rumpf N, Strehl U. Neurofeedback for children with ADHD: a comparison of SCP and theta/ beta protocols. Appl Psychophysiol Biofeedback. 2007;32(2):73-88.

24. Gevensleben H, Kleemeyer M, Rothenberger LG, et al. Neurofeedback in ADHD: further pieces of the puzzle. Brain Topogr. 2014; 27(1):20-32.

25. Sonuga-Barke EJ, Brandeis D, Cortese S, et al; European ADHD Guidelines Group. Nonpharmacological interventions for ADHD: systematic review and meta-analyses of randomized controlled trials of dietary and psychological treatments. Am J Psychiatry. 2013;170(3): 275-289. 
26. Arnold LE, Lofthouse N, Hersch S, et al. EEG neurofeedback for ADHD: double-blind sham-controlled randomized pilot feasibility trial. J Atten Disord. 2013;17(5):410-419.

27. Arns M, de Ridder S, Strehl U, Breteler M, Coenen T. Efficacy of neurofeedback treatment in ADHD: the effects on inattention, impulsivity and hyperactivity: a meta-analysis. Clin EEG Neurosci. 2009;40(3): 180-189.

28. Weiß RH. Grundintelligenztest Skala 2-Revision-(CFT 20-R). Göttingen: Hogrefe; 2006.

29. Petermann F, Petermann U. Wechsler Intelligence Scale for ChildrenFourth Edition (dt. Version) (WISC-IV). Frankfurt/Main: Pearson Assessment; 2011.

30. Melchers P, Preuß U. Kaufman Assessment Battery for Children. Vol 8. Frankfurt/Main: Pearson Assessment; 2009.

31. Hinterberger T, Kaiser J, Kübler A, Neumann N, Birbaumer N. The thought translation device and its applications to the completely paralyzed. In: Diebner H, Druckrey T, Weibel P, editors. Sciences of the Interfaces. Tübingen: Genista-Verlag; 2001:232-240.

32. Hinterberger T. Entwicklung und Optimierung eines Gehirn-ComputerInterfaces mit langsamen Hirnpotentialen. Tübingen: Fakultät für Physik, Eberhard-Karls-Universität Tübingen Schwäbische Verlagsgesellschaft; 1999. [ISBN 3-88466-177-9].

33. Leins U. Neurofeedback für Kinder mit einer Aufmerksamkeitsdefizit-/ Hyperaktivitätsstörung (ADHS). Tübingen: Fakultät für Informations- und Kognitionswissenschaften, Eberhard-Karls-Universität Tübingen; 2004.

34. Zimmermann P, Fimm B, editors. Test for Attentional Performance TAP 1.7. Herzogenrath: Psytest; 2002.

35. de Sonneville L. Amsterdam neuropsychological tasks: a computeraided assessment program. In: Den Brinker BPLM, Beek PJ, Brand AN, Maarse FJ, Mulder LJM, editors. Cognitive Ergonomics, Clinical Assessment and Computer-assisted Learning. Vol Computers in Psychology. Lisse, The Netherlands: Swets \& Zeitlinger; 1999:187-203.

36. Döpfner M, Lehmkuhl G, editors. Diagnostik-System für Psychische Störungen im Kindes- und Jugendalter nach ICD-10 und DSM-IV (DISYPS-KJ). 2. korrigierte und ergänzte ed. Bern: Huber; 2000.
37. Döpfner M, Lehmkuhl G, Steinhausen HC, editors. Kinder-DiagnostikSystem (KIDS), Band 1: Aufmerksamkeitsdefizit- und Hyperaktivitätsstörungen (ADHS). Göttingen: Hogrefe; 2006.

38. Achenbach T. Integrative Guide to the $1991 \mathrm{CBCL} / 4-18$, YSR, and TRF Profiles. Burlington: University of Vermont, Department of Psychiatry; 1991.

39. Arbeitsgruppe Deutsche Child Behavior Checklist. Elternfragebogen über das Verhalten von Kindern und Jugendlichen; deutsche Bearbeitung der Child Behavior Checklist (CBCL 4-18). Einführung und Anleitung zur Auswertung. 2. Auflage mit deutschen Normen. Köln: Arbeitsgruppe Kinder-, Jugend- und Familiendiagnostik (KJFD); 1998.

40. Shapiro SS, Wilk MB. An analysis of variance test for normality (complete samples). Biometrika. 1965;52(3/4):591-611.

41. Arns M, Heinrich H, Strehl U. Evaluation of neurofeedback in ADHD: the long and winding road. Biol Psychol. 2014;95:108-115.

42. Bakhshayesh AR, Hansch S, Wyschkon A, Rezai MJ, Esser G. Neurofeedback in ADHD: a single-blind randomized controlled trial. Eur Child Adolesc Psychiatry. 2011;20(9):481-491.

43. Meisel V, Servera M, Garcia-Banda G, Cardo E, Moreno I. Reprint of "neurofeedback and standard pharmacological intervention in ADHD: a randomized controlled trial with six-month follow-up". Biol Psychol. 2014;95:116-125.

44. Duric NS, Assmus J, Gundersen D, Elgen IB. Neurofeedback for the treatment of children and adolescents with ADHD: a randomized and controlled clinical trial using parental reports. BMC Psychiatry. 2012; 12:107.

45. Faul F, Erdfelder E, Lang A-G, Buchner A. G*Power 3: A flexible statistical power analysis program for the social, behavioral, and biomedical sciences. Behavior Research Methods. 2007;39:175-191.

46. Faul F, Erdfelder E, Buchner A, Lang A-G. Statistical power analyses using $\mathrm{G}^{*}$ Power 3.1: Tests for correlation and regression analyses. Behavior Research Methods. 2009;41:1149-1160.

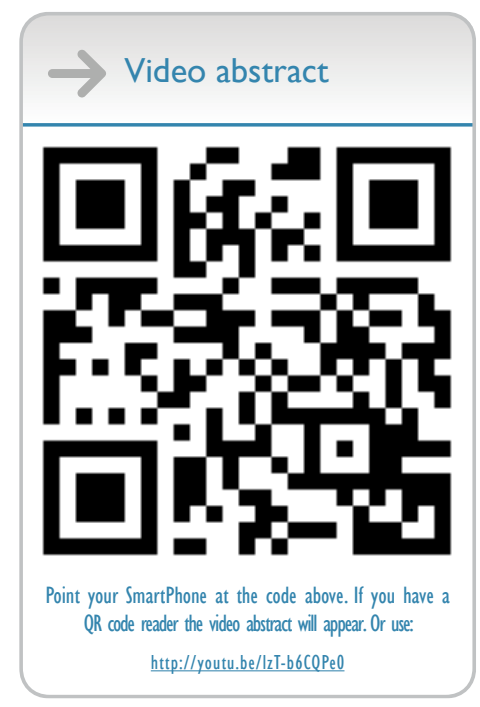

Neuropsychiatric Disease and Treatment

\section{Publish your work in this journal}

Neuropsychiatric Disease and Treatment is an international, peerreviewed journal of clinical therapeutics and pharmacology focusing on concise rapid reporting of clinical or pre-clinical studies on a range of neuropsychiatric and neurological disorders. This journal is indexed on PubMed Central, the 'PsycINFO' database and CAS,

and is the official journal of The International Neuropsychiatric Association (INA). The manuscript management system is completely online and includes a very quick and fair peer-review system, which is all easy to use. Visit http://www.dovepress.com/testimonials.php to read real quotes from published authors. 